\title{
Global familiarity of visual stimuli affects repetition-related neural plasticity but not repetition priming
}

\author{
Anja Soldan, ${ }^{*}$ Eric Zarahn, H. John Hilton, and Yaakov Stern* \\ Cognitive Neuroscience Division of the Taub Institute, Columbia University, USA
}

Received 11 January 2007; revised 6 April 2007; accepted 6 August 2007

Available online 23 August 2007

\begin{abstract}
In this study, we tested the prediction of the component process model of priming [Henson, R.N. (2003). Neuroimaging studies of priming. Prog Neurobiol, 70 (1), 53-81] that repetition priming of familiar and unfamiliar objects produces qualitatively different neural repetition effects. In an fMRI study, subjects viewed four repetitions of familiar objects and globally unfamiliar objects with familiar components. Reliable behavioral priming occurred for both item types across the four presentations and was of a similar magnitude for both stimulus types. The imaging data were analyzed using multivariate linear modeling, which permits explicit testing of the hypothesis that the repetition effects for familiar and unfamiliar objects are qualitatively different (i.e., nonscaled versions of one another). The results showed the presence of two qualitatively different latent spatial patterns of repetition effects from presentation 1 to presentation 4 for familiar and unfamiliar objects, indicating that familiarity with an object's global structural, semantic, or lexical features is an important factor in priming-related neural plasticity. The first latent spatial pattern strongly weighted regions with a similar repetition effect for both item types. The second pattern strongly weighted regions contributing a repetition suppression effect for the familiar objects and repetition enhancement for the unfamiliar objects, particularly the posterior insula, superior temporal gyrus, precentral gyrus, and cingulate cortex. This differential repetition effect might reflect the formation of novel memory representations for the unfamiliar items, which already exist for the familiar objects, consistent with the component process model of priming.

(C) 2007 Elsevier Inc. All rights reserved.
\end{abstract}

Keywords: fMRI; Priming; Multivariate linear models; Objects; Repetition enhancement; Repetition suppression

Repetition priming refers to a behavioral change in the speed, accuracy, or bias of processing a stimulus due to prior exposure to the stimulus (e.g., Tulving and Schacter, 1990). It is a form of

\footnotetext{
* Corresponding authors. Cognitive Neuroscience Division of the Taub Institute, Columbia University, 630 West 168th Street, P \& S Box 16, New York, NY 10032, USA.

E-mail addresses: as1578@columbia.edu (A. Soldan), ys11@columbia.edu (Y. Stern).

Available online on ScienceDirect (www.sciencedirect.com).
}

implicit memory and can occur even when conscious memory for the stimulus is not available (Deeprose and Andrade, 2006; Henson, 2003; Roediger and McDermott, 1993; Schacter and Buckner, 1998; Schacter et al., 1993). Repetition priming is typically measured using indirect tests of memory, which make no explicit reference to the prior encounter with the stimulus. It has been observed both when the same (e.g., Habeck et al., 2006; Henson et al., 2004) or different (Liu and Cooper, 2001; Soldan et al., 2006) tasks are performed during the initial and repeated presentation of a stimulus.

Many neuroimaging studies of repetition priming have demonstrated repetition suppression (RS), a reduction in the neural response to the repeated compared to the first presentation of a stimulus (for reviews, see Henson, 2003; Schacter and Buckner, 1998). These reductions in neural activity tend to occur in a subset of the brain regions that are engaged during the initial analysis of the stimulus. Thus, for visual stimuli, RS tends to be prominent in occipital-temporal cortex, as well as in areas involved in the semantic analysis of stimuli, particularly the inferior frontal gyrus, and is not normally observed in primary visual cortex or in primary motor cortex (Reber et al., 2005; Sayres and Grill-Spector, 2006; Simons et al., 2003; van Turennout et al., 2000; Vuilleumier et al., 2002; Zago et al., 2005). Parallel findings have been demonstrated with single-cell recordings in monkeys, showing a decrease in the neuronal firing rate in inferior temporal cortex and prefrontal cortex for repeated visual stimuli (Desimone, 1996; Ringo, 1996; Sobotka and Ringo, 1994). RS at the neuronal level is stimulusdependent (Sobotka and Ringo, 1994) and for a given stimulus is largest in neurons that showed the greatest response to that stimulus when it was initially presented (Li et al., 1993).

As both repetition priming and RS are repetition-related phenomena and neither depends on explicit memory retrieval, it has been conjectured that RS reflects the neural plasticity that causes repetition priming (Henson, 2003; Maccotta and Buckner, 2004; Schacter and Buckner, 1998; Wiggs and Martin, 1998; Zago et al., 2005). One model for a mechanism by which RS may give rise to priming was proposed by Wiggs and Martin (1998). They suggested that RS in occipital-temporal areas reflects a "sharpening" of the neuronal population representation of a stimulus, such 
that a repeated stimulus can be represented with fewer neurons. When measured with neuroimaging, this would lead to macroscopic RS, as blood flow signal is thought to be a spatial average over several $\mathrm{mm}$ of cortical activity. This more sparse representation is thought to allow for more efficient stimulus processing and hence result in faster behavioral responses (i.e., repetition priming). Others have suggested that the number of neurons representing a repeated stimulus is the same, but their level of activity (GrillSpector et al., 1999) or duration of activity (Henson and Rugg, 2003) is reduced. This is thought to reflect the increased efficiency in the neural processing of a repeated stimulus, which leads to behavioral priming. Going against both models, there have been a few reports of dissociations between repetition priming and RS in occipital-temporal cortex, as measured by fMRI (Dobbins et al., 2004; Sayres and Grill-Spector, 2006). This suggests that not all aspects of RS may be directly related to repetition priming and that further research is necessary to determine the precise relationship between them.

Most neuroimaging studies in support of the view that repetition priming is mediated by RS have used familiar stimuli, such as pictures of everyday objects, animals, famous faces, and words. However, it is still a matter of debate whether repetition priming for familiar and pre-experimentally unfamiliar stimuli, such as nonsense objects, unfamiliar faces, and pseudowords, is mediated by the same neural mechanism, i.e., RS, and the same brain areas. Consistent with the view that RS is a general mechanism that mediates repetition priming of all stimulus types, independent of stimulus familiarity, several studies have demonstrated repetition priming accompanied by RS in occipital-temporal cortex for nonsense (i.e., non-nameable) objects (Habeck et al., 2006; van Turennout et al., 2000; Vuilleumier et al., 2002) and unfamiliar faces (Pourtois et al., 2005). Other studies examining repetition priming of unfamiliar stimuli have reported increases in neural activity, or repetition enhancement (RE), in occipital-temporal cortex for repeated stimuli, including unfamiliar faces (Henson et al., 2000; Thiel et al., 2002), meaningless symbols (Henson et al., 2000), line drawings of novel 3D objects (Schacter et al., 1995), and pseudowords (Fiebach et al., 2005). At least two of these studies (Fiebach et al., 2005; Schacter et al., 1995) only found RE and no RS in occipital-temporal cortex for the unfamiliar stimuli, while Henson et al. (2000) and Thiel et al. (2002) did not report whether there were any regions that showed RS for the unfamiliar stimuli.

Interestingly, both those studies finding RS and those finding RE for unfamiliar stimuli have reported fewer locations of significant neural repetition effects (either RS or RE) for unfamiliar stimuli compared to familiar ones. Thus, those studies reporting RS for both familiar and unfamiliar stimuli within the same task (van Turennout et al., 2000; Vuilleumier et al., 2002) have found that RS for unfamiliar objects was confined to more posterior regions of occipital-temporal cortex, whereas RS for familiar stimuli encompassed both posterior and anterior regions of occipitaltemporal regions as well as inferior frontal regions. Similarly, in the context of repetition priming tasks, RE in occipital-temporal regions for unfamiliar stimuli has been reported in very few and relatively small loci, including the anterior fusiform gyrus (Fiebach et al., 2005; Henson et al., 2000; Schacter et al., 1995; Thiel et al., 2002). In addition, Fiebach et al. (2005) showed that within the same task, familiar stimuli (words) elicited RS in large areas of occipital-temporal cortex, as well as in frontal and parietal regions, whereas unfamiliar stimuli (pseudowords) produced RE in only a subset of occipital-temporal areas.
In order to integrate these findings into a coherent framework, Henson $(2000,2003)$ proposed in his component process model of priming that "repetition suppression occurs whenever the same process is performed on prime [the first presentation of a stimulus] and target [a repeated stimulus], whereas repetition enhancement occurs whenever priming causes a new process to occur on the target that did not occur on the prime" (Henson, 2003, p. 71). With respect to the issue of stimulus familiarity, this model further suggests that RE for unfamiliar stimuli indexes processes related to the formation of new, or abstracted (i.e., high-level) representations, which can occur after a single stimulus presentation. These new high-level representations, in addition to RS-related mechanisms affecting more low-level processes common to both familiar and unfamiliar stimuli, would facilitate stimulus processing on subsequent encounters and thereby contribute to repetition priming effects (Fiebach et al., 2005; Gruber and Müller, 2005). For familiar stimuli, repetition would modulate both lower level and established high-level perceptual and lexical/semantic representations (and hence lead to RS) throughout wide areas of cortex (Henson, 2003), which would all contribute to repetition priming. Note that although the component process model emphasizes anterior occipital-temporal cortex as a location where differential RS/RE effects would be expected for familiar and unfamiliar stimuli, such effects could occur in other brain regions as well, provided that some process occurs for the unfamiliar items that does not occur for the familiar ones. Thus, not only new perceptual, but also semantic or lexical representations might be created for unfamiliar stimuli.

Because the component process model has RS occurring for familiar stimuli, while for unfamiliar stimuli there would be RS in a subset of these regions and RE or no repetition effects in other regions, it predicts that repetition effects for familiar and unfamiliar stimuli should be associated with qualitatively different brain activation patterns. Although past results (Fiebach et al., 2005; Henson et al., 2000; Thiel et al., 2002; van Turennout et al., 2000; Vuilleumier et al., 2002), taken together, suggest that this prediction has been borne out, the imaging analysis approaches they employed (i.e., statistical parametric mapping; SPM) are not appropriate to test it. The reason is that even pure scaling differences between conditions could lead to the existence of true voxel-wise intensity differences between these conditions (see Fig. 1 for an illustration). Likewise, two thresholded SPM maps (one per brain activation pattern) can look quite distinct from each other even when the latent spatial patterns are identical to within a scaling factor. In contrast, a different type of test based on singular value decomposition of brain activation patterns, multivariate linear modeling (MLM), can validly assess whether brain activation patterns are qualitatively different by explicitly determining the number of latent spatial patterns required to summarize them (Worsley et al., 1997; for applications of MLM, see Zarahn et al., 2005, 2007).

We used MLM to test the prediction of the component process model that repetition effects, as measured with BOLD fMRI, associated with line drawings of familiar real-world objects and unfamiliar items have qualitatively different brain activation patterns. Unlike prior studies on this topic, which reported nonsignificant or less priming for the unfamiliar items, the magnitude of priming in this study was comparable for both item types (Hilton et al., 2006), facilitating interpretation of differential neural repetition effects between item types. Furthermore, because the component process model does not differentiate between familiarity for the global object structure and familiarity for local object 


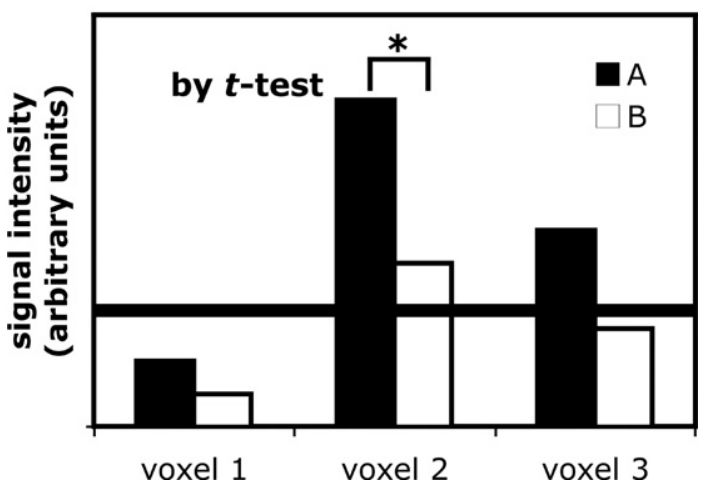

Fig. 1. This example (adapted from Zarahn et al., 2007) illustrates how neither visual inspection of thresholded maps nor condition comparisons via $t$-test formally assesses whether brain activation patterns in two conditions are identical to within a scaling factor or qualitatively different. Conditions $\mathrm{A}$ and $\mathrm{B}$ express identical brain activation patterns, with the expression of this pattern in condition A being twice that in condition B. A visual comparison of thresholded maps between conditions might lead to the incorrect conclusion that brain activation patterns are different in the two conditions because suprathreshold activation in voxel 3 is present in condition $\mathrm{A}$, but not $\mathrm{B}$. When directly comparing voxel-wise intensity differences between conditions via $t$-tests, one might incorrectly conclude that brain activation patterns are different because only voxel 2 tends to be detected as having a difference in intensity between conditions A and B. (*Indicates high probability of detecting a difference between conditions via $t$-test.)

parts or features, we examined whether it is sufficient for objects to be unfamiliar at the global level (while containing familiar local parts) to produce qualitatively different neural repetition effects compared to familiar objects. An effect of global familiarity would suggest that neural plasticity differs as a function of the global structural and/or lexical/semantic representations of objects, not just based on feature or parts representations. The absence of an effect of global familiarity would indicate that unfamiliarity at the global level is not sufficient to affect priming-related neural plasticity and that local unfamiliarity may be necessary. Some of this work has been previously presented as a conference abstract (Zarahn et al., 2004).

\section{Method \\ Subjects}

Fourteen Columbia University students (6 females, 8 males) between the ages of 19 and 29 participated in this study. All reported having normal or corrected-to-normal vision and being free of psychiatric and neurological disorders. Three additional subjects were excluded from the analysis because of data acquisition problems. All subjects gave written informed consent.

Stimuli

Subjects viewed a total of 39 line drawings of real-world (familiar, or F stimuli) objects from the Snodgrass and Vanderwart (1980) stimulus set and 39 line drawings of non-real objects. The non-real stimuli (globally unfamiliar, or U stimuli) consisted of smoothly connected features of real Snodgrass and Vanderwart (1980) objects. See Fig. 2 for examples. Note that although the non-real objects were composed of real parts/local features, the global structure and meaning of the objects were unfamiliar to subjects, as indicated by subjects' fast and highly accurate ability to discriminate the $\mathrm{U}$ from the $\mathrm{F}$ items (see Results). None of the $\mathrm{U}$ objects shared any features with the F objects (i.e., there were two non-overlapping sets of objects).

\section{Procedure}

We used an event-related fMRI design that consisted three blocks, each with a distinct set of $13 \mathrm{~F}$ and $13 \mathrm{U}$ objects. The order of blocks was counterbalanced between subjects. Each block was exactly $8 \mathrm{~min}$ in duration and consisted of 4 buffer trials, followed by an intermixed series of 52 presentations of $\mathrm{F}$ objects (13 objects* 4 presentations/per object) and 52 presentations of $U$ objects. Each object was viewed in one of three different repetition sequences, such that 2, 4, or 6 items intervened between stimulus repetitions. The lag between stimulus repetitions was kept relatively low so as to maximize the likelihood of generating sufficiently robust repetition effects (Henson et al., 2004). The following sequences were used: $[2,4,6],[4,6,2]$, and

\section{Familiar Objects}
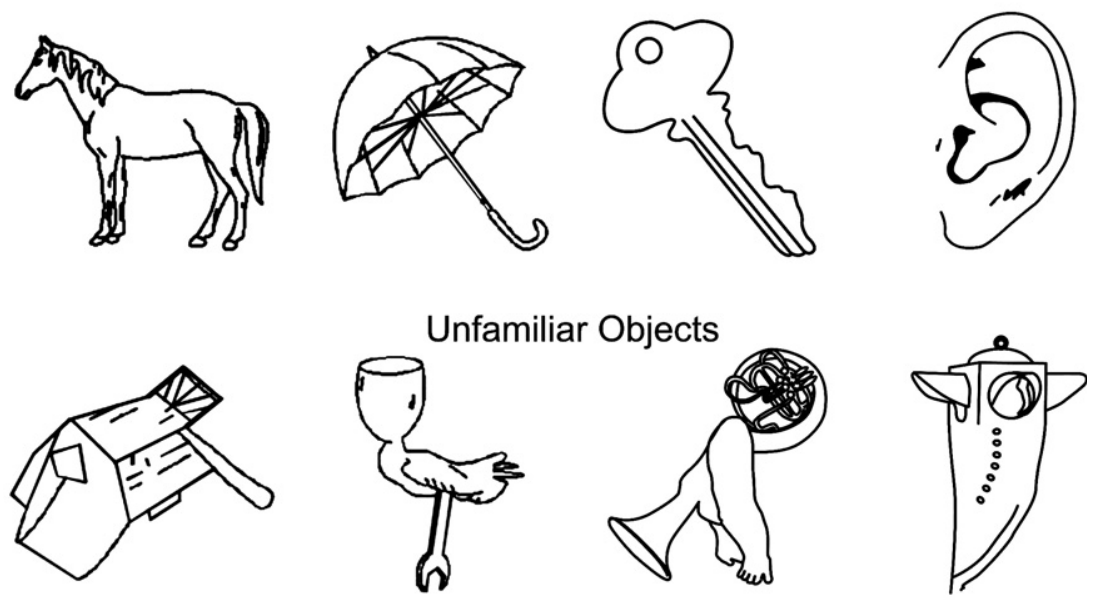

Fig. 2. Examples of the familiar (real) and unfamiliar (non-real) stimuli used in this study. 
$[6,2,4]$. Use of different sequences lowers the predictability of stimuli to participants. The three repetition sequences, which were counterbalanced over stimuli across subjects in a Latin square, were distributed equally throughout the experiment. However, given that 13 objects of each type were presented in a block, each block had 5 repetition sequences of one type and 4 of each of the two others. An additional 52 blank trials were interspersed throughout each block to provide a comparison to baseline and to maintain the required object spacing. During blank trials, the fixation cross was presented, but no stimulus followed and no response was required.

Each trial, including blanks, lasted $3 \mathrm{~s}$ and began with a $500 \mathrm{~ms}$ inter-trial interval (ITI), followed by a $500 \mathrm{~ms}$ fixation cue. Fifty milliseconds after the offset of the fixation cue, the stimulus was presented for $1000 \mathrm{~ms}$. A response interval started coterminously with the stimulus presentation and lasted for $1950 \mathrm{~ms}$. Subjects were asked to indicate via differential button press (LUMItouch button boxes; Photon Control Company) whether a given stimulus depicted a "real" or "non-real" object. This task was thought to encourage global structural processing, as the decision that an object is "nonreal" can only be made if subjects attend to its global shape, not just the components. A right index finger button press was used for real objects and a left index finger press for non-real objects. Both speed and accuracy were emphasized. Task administration and data collection were controlled with PsyScope 1.2.5. All timing was facilitated by the use of the external PsyScope Button Box, which interfaced directly with the PsyScope software, the LUMItouch button boxes, and the MRI acquisition computer. Stimulus presentations were automatically synched to the video retrace signal. Prior to scanning, subjects viewed examples of $F$ and $U$ objects and completed five practice trials. They were not informed that the procedure was a memory test.

\section{fMRI acquisition and preprocessing}

A 1.5 T magnetic resonance scanner (Phillips) was used to acquire functional T2*-weighted images using a gradient echo EPI pulse sequence $\left(\mathrm{TE}=50 \mathrm{~ms} ; \mathrm{TR}=3 \mathrm{sec}\right.$; flip angle $=90^{\circ} ; 64 \times 64$ matrix, $400 \mathrm{~cm}^{2}$ field of view) and a standard quadrature head coil. At the end of the experiment, high resolution (in plane) T2 images were also acquired from each subject at the same slice locations as in the fMRI run using a fast echo spin sequence (TE $=100 \mathrm{~ms}$; $\mathrm{TR}=3 \mathrm{~s} ; 256 \times 256$ matrix; $400 \mathrm{~cm}^{2}$ field of view). Task stimuli were back-projected onto a screen located at the foot of the MRI bed using an LCD projector. Subjects viewed the stimuli via a mirror system located in the head coil.

The data were processed using SPM99 (Wellcome Department of Cognitive Neurology, London) and other code written in Matlab 5.3 (Mathworks, Natick MA). For each subject, images were first corrected for timing of slice acquisition (slice acquisition was ascending, interleaved). All functional volumes were then realigned to the first volume of the first session. The T2 structural image was then co-registered to the first functional volume using the mutual information co-registration algorithm implemented in SPM99. This co-registered high-resolution image was then used to determine parameters $(7 \times 8 \times 7$ non-linear basis functions $)$ for transformation into a Talairach standard space defined by the Montreal Neurological Institute template brain supplied with SPM99. These normalization parameters were then applied to the functional data using sinc interpolation to re-slice the images to $2 \mathrm{~mm} \times 2 \mathrm{~mm} \times 2 \mathrm{~mm}$.

\section{fMRI time-series (i.e., first-level) modeling}

Accuracy was ignored in the imaging analysis, as it was at ceiling for all participants. The regressors for the first-level general linear model were constructed by convolving the default SPM99 hemodynamic response function with the basis functions for each trial type (a rectangular pulse) aligned with stimulus onset. For each subject, the GLM contained 24 regressors, one for each crossing of object type (2), presentation (4), and block (3). Highpass filtering eliminated information below (1/117) Hz. Next, 8 linear contrast images (one for each crossing of object type and presentation, implicitly with respect to baseline) were computed for each subject, averaging across blocks. All contrast images were intensity normalized by dividing each voxel by its time series average, spatially smoothed using a Gaussian kernel of $8 \mathrm{~mm}$ full width-half maximum, masked with an image that had a gray matter prior probability of $>0.25$ (to eliminate ventricles from the search volume), and then used for subsequent second-level (i.e., population-level inference) multivariate analysis. This secondlevel GLM had 8 repeated measures per subject per voxel. Its covariance matrix ( $\Sigma$ in Worsley et al., 1997) was estimated by spatially averaging voxel-wise covariance estimates.

\section{$M L M$}

MLM is an extension of the SPM framework that provides a multivariate test of the spatial null hypothesis that there is no common latent whole brain image pattern of BOLD activation common to two or more contrast images. Like SPM, MLM involves voxel-wise application of the general linear model, but instead of statistically testing for effects of interest (i.e., repetition effects for $\mathrm{F}$ and $\mathrm{U}$ objects) at each voxel, the statistical testing assesses the existence of any such effects simultaneously at all voxels, in other words, in a spatially omnibus fashion. For this reason, MLM will tend to have superior detection power compared with SPM voxel-wise testing in the presence of spatially distributed effects, without inflating Type I error. A second advantage of MLM over SPM is that MLM can explicitly test hypotheses regarding the number of spatial patterns required to summarize the effects of interest. See Fig. 1 for an illustration for why SPM is an ambiguous test of the identity of spatial patterns. MLM does not itself provide localized tests of BOLD contrast values. However, because MLM is an extension of SPM, localization information is easily recovered from the univariate general linear model. Because the goal of this study was to formally test the hypothesis that the spatial patterns of repetition effects, whether RS or RE, are identical for F and $U$ objects, we chose MLM over the more standard SPM approach. However, traditional SPM analyses are also reported (see below).

For the MLM analysis, 2 effects of interest were specified, comprising the contrasts of (1) fMRI signal difference between the 1st and 4th presentation of $F$ objects, and (2) fMRI signal difference between the 1 st and 4 th presentation of $U$ objects. The possible outcomes of an MLM analysis for 2 effects of interest are 0,1 , or 2 latent spatial patterns. In this study, zero latent spatial patterns would indicate that there is no effect of repetition, as assessed by a spatially omnibus null hypothesis. One latent spatial pattern would signify that a main effect of repetition was detected, as assessed by a spatially omnibus null hypothesis, but that there is no interaction between object type and repetition. In other words, the same spatial pattern of brain regions demonstrates repetition 
effects for $\mathrm{F}$ and $\mathrm{U}$ objects. Two latent spatial patterns would indicate that the spatial patterns of repetition effects are not identical for $\mathrm{F}$ and $\mathrm{U}$ objects. In other words, there is an interaction between object type and repetition. Note that the component process model of priming (Henson et al., 2000; Henson, 2003) predicts such an interaction, or the presence of 2 significant latent spatial patterns.

In this study, the effects of interest are said to have dimensionality $k=2$ because two effects of interest were specified. In MLM, singular value decomposition decomposes the $k$-dimensional effects of interest into $k$ latent components (some of which might contain only noise, and others which might contain signal plus noise), each comprising a latent spatial pattern and its latent expression (over conditions in this context). Each latent component is associated with a singular value (or equivalently, an eigenvalue, which is the square of the singular value) that indicates how much variance the latent component explains relative to noise. The first component explains the greatest amount of variance; the second explains the greatest amount of variance after accounting for the first, and so on. To statistically assess the number of latent components containing some signal, a sequential latent root testing procedure is used to compare these eigenvalues to their distribution under noise only (Worsley et al., 1997). Specifically, on the $q$ th step of sequential latent root testing, an $F$-statistic is used to compare the mean of the $q$ th through $k$ th eigenvalue to its distribution under the null hypothesis (which is that there is no signal in any of these components). The procedure stops when $F_{q}<F_{1-\alpha}$, with the inference that $q-1$ components contained signal. The maximum possible number of detected components is always equal to $k$ and the minimum possible number of detected components is always zero. Thus, MLM concerns statistical inference on the number of unique spatial patterns of signal in the effects of interest. In other words, this is the number of linearly independent spatial patterns of signal in the effects of interest, or equivalently the rank of the spatial patterns of signal in the effects of interest.

For this study, the false positive rate of all sequential latent root tests was set to $\alpha=0.05$. The estimated effective spatial degrees of freedom parameter, which may be understood to relate to the number of independent spatial observations, was 423 and the estimated error degrees of freedom at each voxel were 41 . These values are involved in the determination of the degrees of freedom of the $F$-statistics (Worsley et al., 1997); these $F$ degrees of freedom are much larger than those typically encountered in the behavioral sciences because they depend multiplicatively on the spatial degrees of freedom. The latent spatial patterns were thresholded for descriptive and display purposes in all tables and figures at a $t$-value corresponding to $p<0.001$ uncorrected for multiple comparisons and a cluster size of 50 voxels. Anatomic labels for cluster maxima were provided by Talairach Daemon (http://ric.uthscsa.edu/projects/talairachdaemon.html).

\section{SPM analysis}

In order to facilitate the comparison of the present results to previous studies of repetition priming and to directly localize those regions with the strongest contributions to the latent spatial patterns of repetition effects, additional SPM voxel-wise comparisons were performed. The same contrasts analyzed with MLM were queried with SPM: the effect of repetition from presentation 1 to 4 for $F$ and for $\mathrm{U}$ objects, and the interaction between object type and repetition. Contrasts of the parameter estimates from single-subject models were entered into random-effects analyses (two-tailed onesample $t$-tests) comparing the mean parameter estimate over subjects to zero. Results were corrected for multiple comparisons across the whole brain using the Bonferroni correction for the number of resolution elements (resels) in the contrast image (Worsley et al., 1996) and a cluster threshold of $k=5$. This corresponded to a $t$-value of $t=5.6$.

\section{Results}

\section{Behavioral performance}

Preliminary analyses were conducted to examine if nonstimulus-specific practice effects differentially affected priming for $U$ and $F$ items across the three blocks (or scanning sessions). Neither for accuracy nor for RT, there were significant interactions involving the effect of block and presentation (all $p>0.15$ ). Therefore, the data were collapsed across block for all subsequent analyses.

Both the accuracy and RT data were subjected to repeatedmeasures analysis of variance (ANOVA) with object type (F and U) and presentation (1-4) as within-subject factors. Subjects' accuracy was at ceiling for all four presentations of $F$ and $U$ objects and was not affected by presentation or object type (all $p>0.09$; see Fig. 3, bottom panel). As can be seen in Fig. 3, top panel, subjects responded significantly faster to $\mathrm{F}(M=539 \mathrm{~ms}$, $\mathrm{SD}=38 \mathrm{~ms})$ than $\mathrm{U}(M=604 \mathrm{~ms}, \mathrm{SD}=52 \mathrm{~ms})$ objects $[F(1,13)=$ 41.43, $p<0.0001]$. In addition, for RT there was a main effect of presentation $[F(3,39)=169.22, p<0.0001]$ and an interaction between object type and presentation $[F(3,39)=5.78, p=0.012$, Greenhouse-Geisser corrected]. Post hoc profile contrasts in-
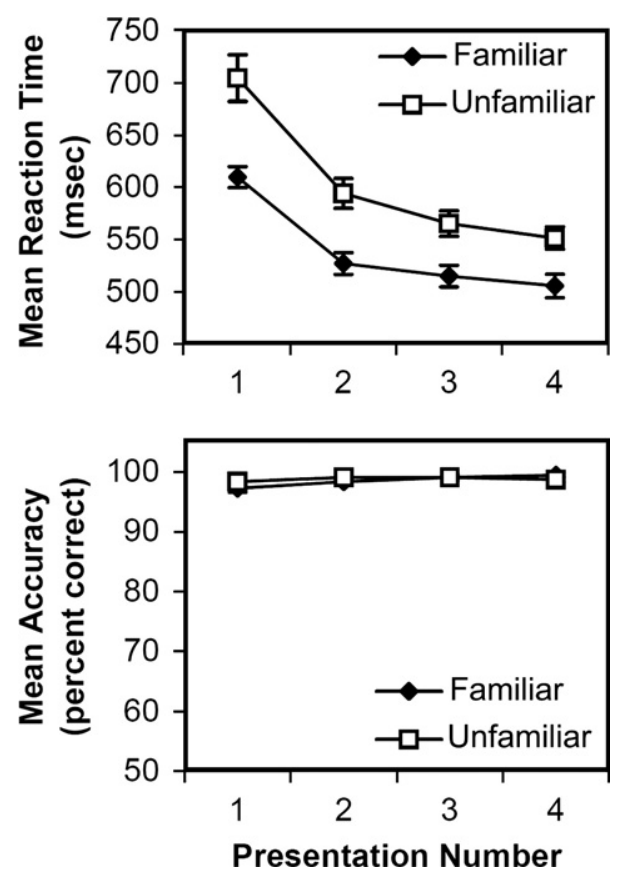

Fig. 3. Behavioral results. Object-decision performance as a function of presentation for familiar and unfamiliar stimuli: mean classification accuracy (top panel) and reaction time (bottom panel). Error bars represent the standard error of the mean. 
dicated that RTs, collapsed across object type, declined with each repetition (from presentation 1 to presentation $2[F(1,13)=191.65$, $p<0.0001]$, presentation 2 to presentation $3[F(1,13)=20.13$, $p \mathrm{~s}<0.001]$, and presentation 3 to presentation $4,[F(1,13)=12.91$, $p<0.005]$ ). Separate post hoc $t$-test for $\mathrm{F}$ and $\mathrm{U}$ items also indicated significant priming from presentation 1 to 4 for both $\mathrm{F}$ objects $[t(13)=14.01, p<0.0001]$ and $U$ objects $[t(13)=9.10$, $p<0.0001]$. The interaction between object type and presentation reflected the fact that there was slightly more priming for $U$ than $\mathrm{F}$ items at presentation $4[t(13)=2.69, p<0.05]$, but not at presentation $2[p=0.1]$.

Because of the baseline difference in RT for F and $\mathrm{U}$ items, not only absolute, but also proportional priming scores (i.e., percentage decrease in RT from presentation 1 to presentations 2, 3, and 4) were computed to assess the validity of the interaction between presentation and object type. An ANOVA with object type and presentation ( 2 vs. 3 vs. 4 ) as factors showed that once baseline differences in RT were taken into account, there was an effect of presentation on $\mathrm{RT}[F(2,26)=29.50, p<0.0001]$, but no interaction between presentation and object type $[F(2,26)=1.36, p=0.27]$. This indicates that proportional priming across repeated presentations was equivalent for $\mathrm{F}$ and $\mathrm{U}$ objects.

\section{fMRI results-MLM analysis}

There were two significant spatial patterns for the effects of interest representing the difference in fMRI amplitude between the 1st and 4th presentation of $\mathrm{F}$ and $\mathrm{U}$ objects [test for one or more spatial components: $F(846,7766)=2.80, p<0.0001$; test for two components: $F(423,5191)=2.25, p<0.0001]$. This means that the brain activation patterns corresponding to these two effects are not simply scaled versions of one another, and so are qualitatively different. The eigenvalues for the first and second latent patterns were 3.53 and 2.36, respectively. After accounting for noise (by subtracting 1 from each eigenvalue), the first latent pattern accounted for approximately $65 \%$ and the second pattern for $35 \%$ of the total signal variance in the effects of interest.

In interpreting latent spatial patterns, it is important to understand that the net activation across conditions (in the effects of interest) is equal to a weighted sum of the latent patterns. Therefore, the latent patterns are to be understood as contributions to the net activation patterns. The same applies to the voxel-level, where each latent component makes a contribution to the net voxel activation across conditions. The first latent spatial pattern had negative $^{1}$ expression, in terms of presentation 1 minus presentation 4 , for both object types. Therefore, at each spatial location, it reflects same-signed contributions to RS (for negative spatial weights) or RE (for positive spatial weights) for both $\mathrm{F}$ and $\mathrm{U}$ object types. However, the magnitude of expression was approximately $120 \%$ greater for $U$ than $\mathrm{F}$ objects, which means that a given spatial weight implies a greater contribution to RS/RE for $\mathrm{U}$ than $\mathrm{F}$ objects (see Fig. 4). The first latent pattern, thresholded for descriptive purposes, is depicted in Fig. 5. Many of those regions with strong RS contributions have previously been reported to show RS in repetition priming tasks (Dobbins et al.,

\footnotetext{
${ }^{1}$ The signs of the spatial weights and the signs of their corresponding expressions in isolation are arbitrary and only meaningful in their product. To determine the contribution a latent spatial pattern makes to the activation over conditions at any given voxel, one multiplies the signed weight of the latent spatial pattern at that voxel by its signed expression.
}

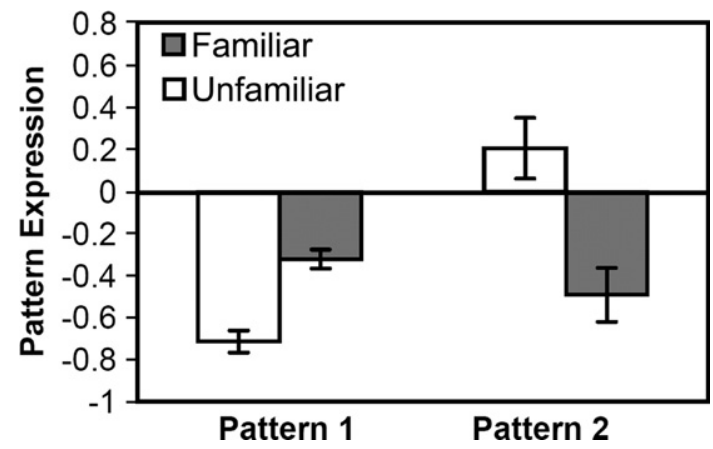

Fig. 4. Predicted expressions of scaled latent spatial patterns for familiar and unfamiliar objects for the effects representing the difference in fMRI amplitude from the 1 st to the 4 th presentation of familiar and unfamiliar objects.

2004; Habeck et al., 2006; Maccotta and Buckner, 2004; Simons et al., 2003). See Table 1 for a list of RS and RE contribution maxima and Fig. 6 for a horizontal slice through occipital-temporal cortex showing regions with a large RS contribution to latent spatial pattern 1. The second latent spatial pattern reflected oppositely signed activation repetition effects for $F$ and $U$ object types, as the signs of pattern expression were opposite for $F$ and $U$ objects (see Fig. 4). Since the weights of latent pattern 2 were all negative, in strongly weighted regions, this latent pattern contributed RS for F objects and RE for $U$ objects (Table 1). Notably, no areas in occipital-temporal cortex were weighted strongly in this pattern.

The goal of the above MLM analysis was to statistically assess if brain activation patterns of repetition-related fMRI signal change for $\mathrm{F}$ and $\mathrm{U}$ stimuli are qualitatively different (they were), not per se to identify areas exhibiting RS or RE. As the net activation change at each voxel is a weighted sum of contributions from all of the latent components, one needs to be careful to not simply assume that the activation at a given voxel looks exactly like the expression of a latent spatial pattern for which it might be heavily weighted. For descriptive and hypothesis generation purposes, we therefore examined how activity at individual locations heavily weighted in the MLM latent patterns actually changed across the four presentations. In particular, it was important to assess whether decreases or increases in fMRI response amplitude do indeed reflect modulation (either suppression or enhancement) of an initial positive response (as opposed to a negative one) with respect to baseline on presentation 1 .

For regions with the strongest negative spatial weights for latent pattern 1, the magnitude of RS from presentation 1 to 4 was very similar for $\mathrm{F}$ and $\mathrm{U}$ objects; however, the rate of RS across the intervening presentations was evidently different (Fig. 5). Whereas F objects showed large RS from presentation 1 to 2 and very little change with subsequent presentations, $U$ objects showed a more gradual decline in activation with each presentation. By contrast, areas showing fMRI signal increases from presentation 1 to 4 (i.e., those with positive weights for latent spatial pattern 1) showed a similarly linear increase across presentations for both types of objects, although the overall amount of change was greater for $U$ than for F objects. Importantly, however, these increases in fMRI signal amplitude did not reflect true RE, but rather decreases in deactivation relative to baseline.

Voxels strongly expressing latent spatial pattern 2 showed a gradual and fairly linear decline in activation for $\mathrm{F}$ objects with 
each presentation and no change or a gradual increase in activation for $U$ objects (see Fig. 7). This activation increase for $U$ objects represented RE of an initially positive response with respect to baseline at certain brain regions, including the precentral gyrus, left posterior insula, and left cingulate gyrus (data not shown), but not in occipital-temporal cortex, even at a lower statistical threshold

a

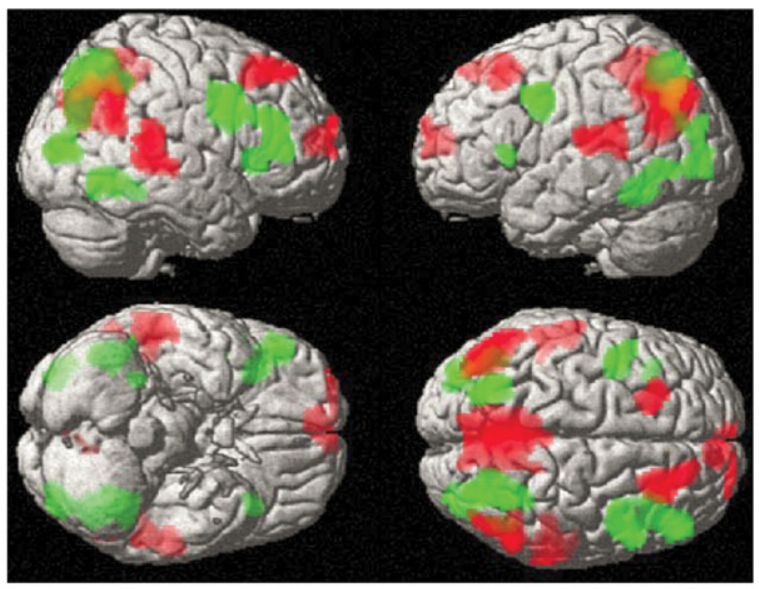

\section{Green: Repetition Suppression}

\section{Red: Repetition Enhancement}

b.i

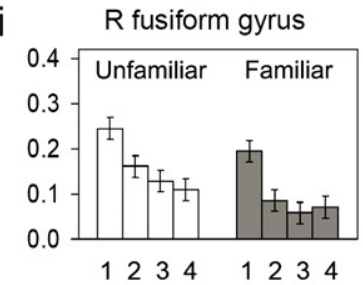

c.i

$R$ medial precuneus

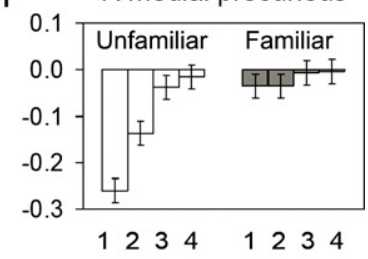

b.ii

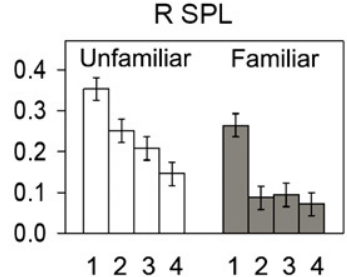

c.ii

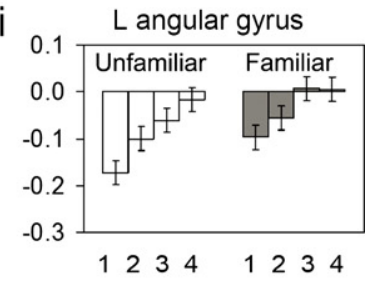

b.iii

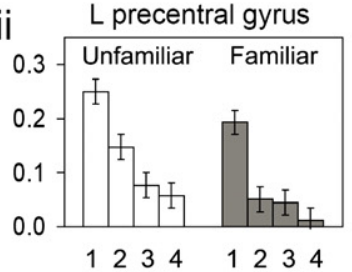

C.iii

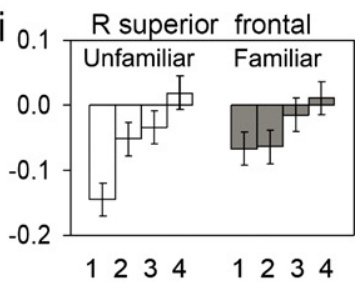

b.iv L anterior insula

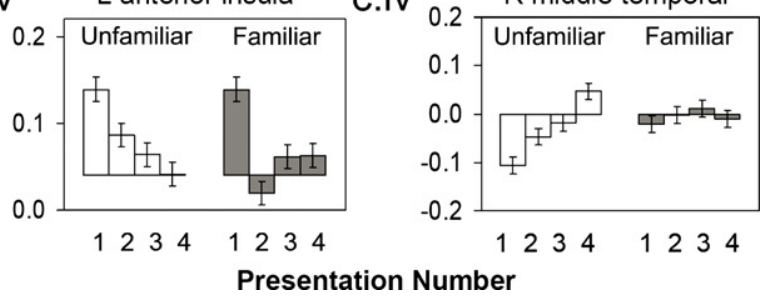

$(p<0.005$, cluster size $=10$ voxels). Other regions (e.g., postcentral gyrus, superior temporal gyrus) initially showed a negative response with respect to baseline that became more positive over presentations.

\section{SPM results}

Several of the brain regions that were strongly weighted in latent pattern 1 demonstrated a main effect of repetition (see Table 2 ). There were two regions that showed a significant interaction between repetition and object type: the right medial precuneus (BA $7, x=-8, y=-48, z=50, k=7$ voxels), and the left posterior insula (BA 13, $x=-34, y=-15, z=14, k=22$ voxels). The precuneus region showed $\mathrm{RE}$ of an initially negative response with respect to baseline for $\mathrm{U}$ objects and a non-significant RE effect for F objects. This region had large positive spatial weights in spatial pattern 1 , which heavily weighted regions with a similar RS/RE effect for both object types. The left insula region, by comparison, was heavily weighted in latent spatial pattern 2; it showed RE of an initially negative response for $U$ objects and RS of an initially positive response for $\mathrm{F}$ objects. At a lower statistical threshold ( $p<0.001$ uncorrected, $k=5$ voxels), additional regions were detected that demonstrated an interaction, although none was located in the occipital-temporal cortex. Some of these regions were strongly weighted in spatial pattern 1 , some in pattern 2 , thus demonstrating the usefulness of MLM in differentiating between brain regions that show qualitatively different repetition effects and regions that show qualitatively identical effects, but at a different magnitude.

When $\mathrm{U}$ and $\mathrm{F}$ objects were considered separately, significant RS was present in several brain regions for both object types (see Table 2), but significant RE was only detected for U objects, even when the threshold was lowered to $p=0.001$ uncorrected. Thus, based on the SPM results alone, one might conclude that RE is a process that only occurs for $U$ objects. The MLM analysis, however, showed that $\mathrm{F}$ objects also contributed an RE effect to spatial pattern 1 in the same regions as $U$ objects, just at a lower magnitude.

\section{Discussion}

This study showed that, in spite of producing very similar levels of repetition priming, a behavioral effect, the brain patterns of

Fig. 5. (a) First latent spatial pattern, scaled by its singular value, reflecting similar contributions to RS/RE for both familiar and unfamiliar objects for the effect representing the difference in fMRI amplitude between presentation 1 and 4. Positive voxel weights (i.e., those showing RE) are displayed in red; negative voxel weights (i.e., those showing RS) are displayed in green. (b) Rate of repetition suppression and enhancement. Graphs show mean fMRI signal change (on $y$-axis) at voxels strongly expressing the first spatial pattern as a function of presentation number (on $x$-axis). Displayed voxels represent cluster maxima for the four regions with the highest pattern expression. Voxels from only one hemisphere are shown as the data were very similar in the corresponding region of the other hemisphere. Left panel shows differential rate of repetition suppression for familiar and unfamiliar objects across the four presentations in right fusiform gyrus (i), right superior parietal lobule (ii), left precentral gyrus (iii), and left anterior insula (iv). Right panel shows similar rate of signal increase (reduction in task-induced deactivation) for familiar and unfamiliar objects in right medial precuneus (i), left angular gyrus (ii), right superior frontal gyrus (iii), and right middle temporal gyrus (iv). 
Table 1

Regions with strong RS and RE contributions to latent spatial patterns 1 and 2

\begin{tabular}{l} 
Structure \\
\hline Pattern 1, positive weights \\
R medial frontal gyrus \\
R middle frontal gyrus \\
L middle frontal gyrus \\
R superior frontal gyrus \\
L superior frontal gyrus \\
R precuneus \\
L precuneus \\
L angular gyrus \\
R inferior parietal lobule \\
R middle temporal gyrus \\
R superior temporal gyrus \\
L superior temporal gyrus \\
R posterior insula \\
L posterior insula
\end{tabular}

Pattern 1, negative weights

$\mathrm{R}$ inferior frontal gyrus

L inferior frontal gyrus

$\mathrm{R}$ middle frontal gyrus

$\mathrm{L}$ precentral gyrus

$\mathrm{R}$ middle occipital gyrus

L middle occipital gyrus

$\mathrm{R}$ fusiform gyrus

$\mathrm{L}$ fusiform gyrus

$\mathrm{L}$ middle temporal gyrus

$\mathrm{R}$ precuneus

$\mathrm{R}$ superior parietal lobule

L superior parietal lobule

$\mathrm{R}$ anterior insula

$\mathrm{L}$ anterior insula

BA

$x$

$y$

$z$

$z$-score

$\begin{array}{lrrrl}10 & 12 & 55 & 10 & 4.41^{*} \\ 8 & 30 & 21 & 39 & 4.48^{*} \\ 8 & -26 & 24 & 43 & 4.39^{*} \\ 8,10 & 24 & 33 & 46 & 5.13 \\ 6 & -18 & 24 & 50 & 4.21^{*} \\ 7 & 8 & -55 & 34 & 6.39 \\ 39 & -42 & -70 & 37 & 6.38 \\ 39 & -53 & -62 & 34 & 5.46 \\ 40 & 51 & -51 & 36 & 4.71 \\ 21 & 55 & -31 & 2 & 5.25 \\ 22 & 63 & -32 & 15 & 4.48^{*} \\ 40 & -57 & -23 & 12 & 4.15^{*} \\ 13 & 46 & -32 & 20 & 4.79 \\ 13 & -51 & -34 & 18 & 4.09^{*}\end{array}$

$\begin{array}{lrrrl}9,46 & 50 & 35 & 7 & 6.56 \\ 9 & -42 & 3 & 29 & 5.90 \\ 46 & 48 & 34 & 17 & 5.76 \\ 6 & -44 & 4 & 35 & 6.98 \\ 19 & 36 & -84 & 6 & 4.46^{*} \\ 19 & -32 & -82 & 2 & 4.46^{*} \\ 37 & 44 & -50 & -8 & 4.79 \\ 37 & -38 & -52 & -12 & 4.90 \\ 37 & -40 & -56 & -1 & 5.03 \\ 7 & 24 & -60 & 51 & 5.46 \\ 7 & 30 & -68 & 48 & 5.74 \\ 7 & -24 & -66 & 44 & 5.68 \\ 13 & 34 & 22 & 2 & 4.86 \\ 13 & -36 & 20 & 5 & 5.40\end{array}$

Pattern 1, negative weights

$\mathrm{R}$ inferior frontal gyrus

$\mathrm{R}$ middle frontal gyrus

$\mathrm{R}$ precentral gyrus

$\mathrm{L}$ precentral gyrus

$\mathrm{R}$ superior temporal gyrus

L superior temporal gyrus

$\mathrm{R}$ postcentral gyrus

L postcentral gyrus

$\mathrm{L}$ posterior insula

L cingulate gyrus

\begin{tabular}{lrrrl}
45 & 40 & 23 & 3 & $4.24^{*}$ \\
46 & 44 & 36 & 20 & $4.21^{*}$ \\
4 & 61 & -10 & 30 & $3.96^{*}$ \\
6,9 & -59 & -4 & 28 & $4.22^{*}$ \\
41,42 & 63 & -32 & 16 & $4.51^{*}$ \\
41 & -46 & -27 & 7 & $4.17^{*}$ \\
5 & 38 & -47 & 61 & $3.91^{*}$ \\
3,5 & -22 & -34 & 57 & 5.29 \\
13 & -38 & -7 & 15 & 5.47 \\
24 & -4 & -2 & 35 & $4.05^{*}$ \\
\hline
\end{tabular}

The nearest gray-matter voxel locations of positive and negative weights in the latent spatial patterns representing the repetition effect from presentation 1 to 4 for familiar (F) and unfamiliar (U) objects are listed (activation peaks significant at $p<0.05$ corrected for multiple comparisons, except for $* p<0.001$ uncorrected, cluster size $=50$ voxels). Pattern 1 positive weights correspond to regions contributing an increase in activation with stimulus repetition for both $\mathrm{F}$ and $\mathrm{U}$ objects; negative weights denote areas that contribute a decrease in activation with stimulus repetition for both $\mathrm{F}$ and $\mathrm{U}$ objects. Pattern 2 negative weights correspond to regions contributing a decrease in activation for $\mathrm{F}$ objects and an increase in activation for $\mathrm{U}$ objects.

repetition effects of globally familiar stimuli and stimuli with preexperimentally unfamiliar global structure are qualitatively different. This latter finding is of significance because it indicates that familiarity with an object's global structural, semantic, and/or lexical features is an important factor in neural plasticity. Previous imaging studies using unfamiliar objects used stimuli without familiar local features.

The inference of a qualitative dependence of repetition effects on global stimulus familiarity was obtained from the MLM result of two significant latent patterns associated with these repetition effects. Previous studies have not been able to unambiguously assess this hypothesis due to the ambiguity of SPM tests with respect to the (alternative) hypothesis of qualitatively different brain activation patterns. Thus, unlike most imaging studies, the formal result here is not where an effect of interest is detected in the brain but rather the number of patterns of brain activation required to explain the effects of interest (Worsley et al., 1997).

Still, the net activation at any voxel (within a given set of effects of interest) is a weighted sum of the contributions from the latent patterns and it is useful for hypothesis generation and speculative purposes to consider these patterns descriptively. Latent spatial pattern 1 tended to weight heavily brain areas with a similar repetition effect on the neural responses of both object types. Some brain regions contributed a strong RS effect to latent pattern 1, whereas other regions contributed a task-induced deactivation (TID) effect to latent spatial pattern 1 for both $\mathrm{F}$ and $U$ objects. In contrast, latent spatial pattern 2 tended to weight heavily brain areas with RS for F objects and RE for U objects. These results are broadly consistent with Henson's (2003) component process model of priming, which predicted one network of brain regions where RS would occur for both $\mathrm{F}$ and U objects (latent spatial pattern 1), as well as a second network where RS would occur for F objects and RE or no repetition effects would be present for U objects (latent spatial pattern 2).

Those brain regions contributing a large RS effect for both $\mathrm{F}$ and U objects (latent spatial pattern 1) were located in occipitaltemporal cortex, inferior frontal regions, and parts of superior parietal cortex. The RS effects in occipital-temporal and inferior frontal cortex may reflect facilitation in the visual and semantic analysis of the objects, respectively, consistent with many prior studies of repetition priming (Habeck et al., 2006; Henson et al., 2004; Maccotta and Buckner, 2004; Reber et al., 2005; Simons et al., 2003; Vuilleumier et al., 2002). Interestingly, the presence of familiar object parts was sufficient to activate and then modulate

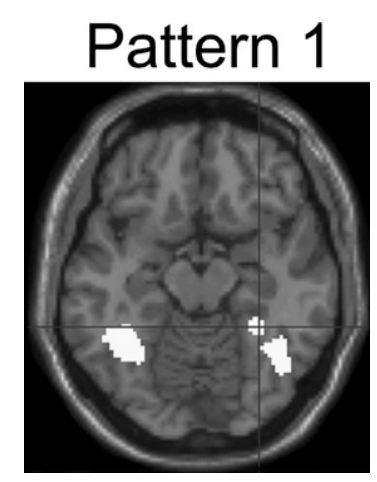

Fig. 6. Horizontal slice through latent spatial pattern 1 showing voxels with large negative weights in mid-to anterior bilateral fusiform gyri (local maximum shown $=32,-42,-14$ in $\mathrm{mm}$ in MNI standard brain space). The slice illustrates the similar contribution to the RS effect for both familiar and unfamiliar objects for the effect representing the difference in fMRI amplitude between presentation 1 and 4 . Note that although the overall RS/ RE contribution to pattern 1 was greater for $U$ than $F$ objects, this does not imply that the RS/RE contribution to pattern 1 in any given spatial location is greater for $\mathrm{U}$ than $\mathrm{F}$ objects (see Results). 
a

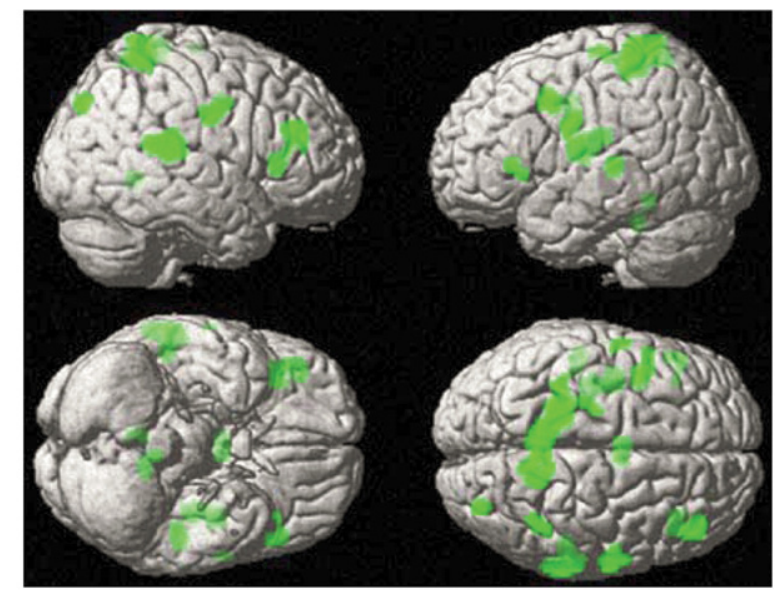

Repetition Suppression - real objects, Repetition Enhancement - non-real objects

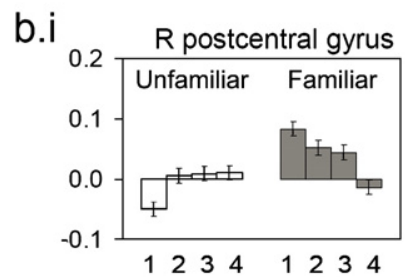

b.i

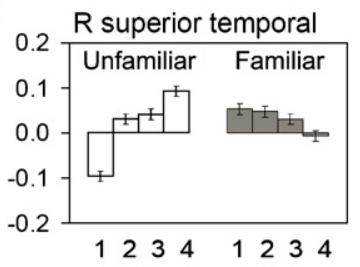

b.ii

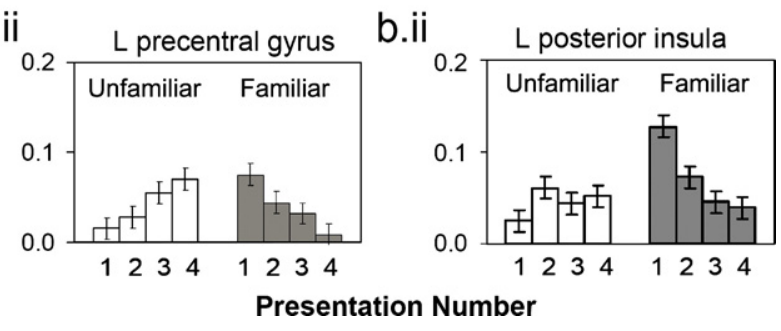

Fig. 7. (a) Second latent spatial pattern, scaled by its singular value, representing regions with opposite contributions to the effect of stimulus repetition from presentations 1 to 4, namely RS for familiar objects and RE for non-real objects. (b) Graphs show mean fMRI signal change (on $y$-axis) at four cluster maxima with the strongest weights for the second spatial pattern as a function of presentation number (on $x$-axis) in the right postcentral gyrus (i), left precentral gyrus (ii), right superior temporal gyrus (iii), and left posterior insula (iv).

these visual and semantic representations. Although it is also possible that the familiar parts of the $U$ objects activated representations pertaining to whole objects in these regions, consistent with some prior findings (Lerner et al., 2001), the slower rate of RS indicates that neural plasticity in these regions did depend on global perceptual and semantic stimulus properties.

The strong RS contribution to both $\mathrm{F}$ and $\mathrm{U}$ objects in the superior parietal lobule may signify a reduction in the amount of motor attention necessary to prepare the correct motor response for the repeated stimuli. Thus, superior parietal cortex has been shown to be involved in sensory-motor integration (Andersen et al., 1997; Iacoboni et al., 1998; Tanabe et al., 2005) and motor attention (i.e., covert preparation of an upcoming movement, switching intended movements, hand-eye coordination) (Astafiev et al., 2003; Battaglia-Mayer and Caminiti, 2002; Rushworth et al., 2003). Consistent with this idea, large RS was also found in premotor cortex (BA 6), a region that is important for planning movements and that receives strong inputs from the superior parietal lobule (Schubotz and von Cramon, 2003; Wise et al., 1997). The presence of large RS effects in the superior parietal lobule and premotor cortex is consistent with the view that priming may not only reflect more efficient processing of the perceptual and semantic properties of stimuli, but also greater efficiency in linking a particular decision (Schnyer et al., 2006) and/or motor response (Dobbins et al., 2004) to a repeated stimulus. This interpretation is consistent with recent findings showing a correlation between repetition priming and RS in precentral gyrus (Orfanidou et al., 2006) and superior parietal lobule (Habeck et al., 2006).

The notion that the repetition priming effects in this study partially reflected greater automatization in generating the correct decision and/or response to a repeated compared to new stimulus is bolstered by the repetition-induced changes in task-induced deactivation (TID) for $\mathrm{F}$ and $\mathrm{U}$ objects (i.e., those with positive weights in latent spatial pattern 1). These regions deactivated during task performance, such that fMRI activity was lower when stimuli were presented than during baseline. The magnitude of this deactivation, however, gradually decreased across stimulus repetitions for both object types, and by the fourth stimulus presentation, it was at or close to zero (i.e., baseline). Although deactivation in this network was observed for both object types, it occurred more strongly for $\mathrm{U}$ than for $\mathrm{F}$ objects. TID has been observed in several of these regions in other experimental paradigms and is thought to occur independently of the specific experimental task (Binder et al., 1999; Gould et al., 2006; McKiernan et al., 2003). The set of regions showing TID across tasks is also referred to as the default network (Raichle et al., 2001).

It is currently postulated that TID reflects the reallocation of processing resources from areas not directly involved in task performance to areas that are necessary for task performance (Gusnard and Raichle, 2001; McKiernan et al., 2003). It has also been similarly claimed that TID may in part result from the suspension of spontaneous semantic activity that occurs during rest, or the "stream of consciousness" that is interrupted during task performance (McKiernan et al., 2006). Given that TID has been shown to increase with task-difficulty (McKiernan et al., 2003), the greater TID for the first presentation of $U$ than $F$ objects in the present study could reflect the greater difficulty, or time required for processing $U$ stimuli compared to $F$ ones. Furthermore, the decrease in TID as a function of stimulus repetition may indicate that performing the same task on repeated stimuli became less difficult for subjects, or was associated with less processing time. This interpretation goes hand-in-hand with the proposal that other aspects of task performance were facilitated by stimulus repetition, as reflected by task-related RS in many brain regions. In other words, TID may be inversely related to task automaticity, such that the first time a decision has to be made for a particular stimulus, processing demands are high, as indicated by high TID levels and high activation levels in regions engaged in stimulus analysis and task performance. When the same decision is repeatedly made for the same stimulus, processing demands are decreased, thus reducing both TID and task-related activation. A less likely possibility is that changes in TID are actually directly related to repetition priming, such that increased activity in these regions somehow facilitates performance of the object-decision task.

The view that repetition priming results from facilitation at several different levels of task performance (perceptual, semantic, decision, response) is consistent with the component process model 
Table 2

Regions showing repetition suppression or enhancement effects, as assessed by SPM

\begin{tabular}{llrrrr}
\hline Structure & BA & $x$ & $y$ & $z$ & $z$-score \\
\hline Repetition suppression common for familiar and & unfamiliar objects \\
R inferior frontal gyrus & 9,45 & 36 & 27 & 6 & 4.41 \\
R middle frontal gyrus & 46 & 46 & 30 & 17 & 4.59 \\
R precentral gyrus & 6 & 38 & 3 & 27 & 4.07 \\
L fusiform gyrus & 37 & -36 & -55 & -8 & 4.29 \\
R superior parietal lobule & 7 & 22 & -61 & 53 & 4.31 \\
& & & & & \\
Repetition suppression for familiar objects & & & & \\
R inferior frontal gyrus & 46,47 & 34 & 25 & -1 & 4.65 \\
R middle frontal gyrus & 9,46 & 55 & 17 & 27 & 4.47 \\
R middle frontal gyrus & 6 & 40 & 8 & 42 & 4.32 \\
R fusiform gyrus & 37 & 45 & -49 & -8 & 4.24 \\
L inferior temporal gyrus & 19,37 & -42 & -64 & -5 & 4.35 \\
L superior temporal gyrus & 22 & -57 & -4 & 4 & 4.39 \\
R precuneus & 7 & 22 & -60 & 51 & 4.71 \\
R superior parietal lobule & 7 & 28 & -66 & 44 & 4.14 \\
Repetition suppression for unfamiliar objects & & & & \\
R inferior frontal gyrus & 9 & 44 & 3 & 26 & 4.12 \\
L fusiform gyrus & 37 & -38 & -47 & -11 & 4.46 \\
L parahippocampal gyrus & 19 & -32 & -53 & -7 & 4.11 \\
L middle temporal gyrus & 37 & -44 & -62 & 0 & 4.43 \\
R superior parietal lobule & 7 & 22 & -64 & 47 & 4.12 \\
L anterior insula & 13 & -36 & 18 & 1 & 4.50 \\
& & & & &
\end{tabular}

Repetition enhancement common for familiar and unfamiliar objects

$\begin{array}{llllll}\text { Right medial frontal gyrus } & 10 & 0 & 10 & 55 & 4.20\end{array}$

Repetition enhancement for familiar objects

N/A

Repetition enhancement for unfamiliar objects

\begin{tabular}{llrlll} 
L paracentral lobule & 5 & -1 & -42 & 57 & 4.22 \\
R precuneus & 31 & 6 & -53 & 32 & 4.39 \\
L precuneus & 19 & -40 & -72 & 40 & 4.38 \\
R inferior parietal lobule & 40 & 51 & -51 & 36 & 4.55 \\
L angular gyrus & 39 & -51 & -66 & 36 & 4.54 \\
\hline
\end{tabular}

The nearest gray-matter voxel locations of activation peaks significant at $p<0.05$ corrected for multiple comparisons, cluster size $=5$ voxels.

of priming (Henson, 2003). In this regard, it is useful to consider those regions showing distinct neural repetition effects for the $\mathrm{F}$ and $\mathrm{U}$ items in this study (i.e., RS for $\mathrm{F}$ objects and RE for $\mathrm{U}$ objects, latent spatial pattern 2). Regions strongly contributing to this pattern consisted of the left posterior insula, left precentral gyrus, left cingulate gyrus, and bilateral superior temporal gyrus. According to the component process model, RE in these regions reflects a process that did not occur during initial viewing of the $U$ items. The same process, however, did presumably occur for the $\mathrm{F}$ items, as indicated by an RS contribution for these objects.

Repetition-related activation increases of an initially positive response in the left insula have been reported in previous studies of repetition priming (Reber et al., 2005; van Turennout et al., 2003; van Turennout et al., 2000). Unlike the present study, these RE effects were only found for F objects. According to van Turennout et al. (2000), these increases in left insula activity may index the strengthening of the association between a picture of an object and its lexical representation. Although the task in the current experiment was to classify the pictures as 'real' or 'non-real', subject may have covertly named or attempted to name the figures. Thus, RE in the left insula for $\mathrm{U}$ objects may reflect increased lexical processing for repeated $U$ objects, as subjects were trying to generate appropriate verbal labels for the $\mathrm{U}$ items. RS for F objects, by comparison, could index facilitated access to the lexical representation of repeated $\mathrm{F}$ objects. A similar argument could be made for activation in the superior temporal cortex and the left inferior precentral gyrus, which also showed strong RE contributions for $U$ objects and RS for F objects. Thus, the superior temporal cortex has been shown to be involved in the perception and production of language (Buchsbaum et al., 2001) and object naming (Hirsch et al., 2001). Similarly, the area in left inferior precentral gyrus (BA 6), extending into the inferior frontal gyrus (BA 9) has been proposed to be involved in articulatory processes (Pulvermüller et al., 2006) and sub-vocalization activity (Dietz et al., 2005), as well as in certain naming and word-retrieval tasks (Etard et al., 2000; Grabowski et al., 1998). Therefore, like the left insula, these regions may be involved in lexical retrieval or covert naming of the $F$ and $U$ objects. This interpretation would be consistent with the component process model's proposal that RE reflects the formation of new memory representations. Importantly, this study also demonstrated that RE effects for $\mathrm{U}$ stimuli need not be of a perceptual nature, as suggested by prior studies (Fiebach et al., 2005; Henson et al., 2000; Schacter et al., 1995; Thiel et al., 2002), but may reflect the learning of more abstract (i.e., lexical) information. It is unclear, however, from the current data set, whether these RE (and RS) effects directly contributed to the observed behavioral priming effects or simply co-occurred with them.

Another, though unlikely possibility is that the differential patterns of repetition effects for $U$ and $F$ items reflect differential changes in eye movements from presentation 1 to 4 for the two items types. Given that none of the regions that showed differential repetition effects as a function of stimulus type is known to play a dominant role in the generation of eye movements (Krautzlis, 2005), we believe that eye movements cannot account for the present results.

Previous studies reporting RE effects for $\mathrm{U}$ items noted these effects in occipital-temporal regions, whereas in this study, no regions were detected that contributed an $R E$ effect for $U$ items in the occipital-temporal cortex, even at a lenient statistical threshold (i.e., latent spatial pattern 2). This may indicate that no new global structural/perceptual representations were formed for the $U$ objects, despite three repetitions of each item. One possibility is that subjects may have been attempting to segment the $U$ objects into identifiable parts, rather than form a unified percept of the conjunction of these parts. These part representations or even the representations of the whole component objects then gave rise to $\mathrm{RS}$ in the occipital-temporal cortex. Alternatively, it is also possible that the formation of new perceptual representations is not necessarily accompanied by RE, but that some form of neural sharpening can take place even for the first repetition of a previously unfamiliar object, leading to RS only. Given that the U objects contained familiar parts, this type of rapid perceptual learning might have occurred in this study.

On a more general note, the lack of an interaction between object type and presentation in occipital-temporal regions suggests that objects must be unfamiliar at both the global and the local level in order to affect the spatial pattern of neural plasticity in object-sensitive cortex. This finding has implications for prior studies that have postulated a laterality difference in the functional organization of occipital-temporal cortex, particularly fusiform 
gyrus (Koutstaal et al., 2001; Marsolek, 1999; Simons et al., 2003; Vuilleumier et al., 2002). In this view, the left fusiform is specialized for representing more abstract visual form and lexical/ semantic information and shows neural plasticity only for $\mathrm{F}$ objects, whereas the right fusiform gyrus is specialized for representing form-specific visual properties and shows neural plasticity for both F and U objects (Fiebach et al., 2005; Schacter et al., 1995; Vuilleumier et al., 2002). In the present study, the dominant latent spatial pattern contributed a similar RS effect for both object types in left and right fusiform gyrus for $F$ and $U$ objects (Fig. 6). These results suggest that the left fusiform specificity for $\mathrm{F}$ objects is restricted to objects that are unfamiliar at both the global and the local level. At the same time, the differential rate of RS in occipital-temporal cortex indicates that perceptual learning occurred more slowly for the globally $U$ than the $\mathrm{F}$ items. This finding might explain why some prior studies using only a single stimulus repetition reported smaller (in terms of magnitude spatial extent) RS effects for $\mathrm{F}$ than $\mathrm{U}$ items.

A final aspect of the current results that is noteworthy concerns the relationship between the magnitude of behavioral priming and the magnitude of the dominant (in terms of variance explained) RS effects (latent spatial pattern 1). Behaviorally, there was equivalent proportional priming for the $\mathrm{F}$ and $\mathrm{U}$ objects, whereas neurally, the rate of RS differed for the $\mathrm{F}$ and $\mathrm{U}$ objects, with $\mathrm{F}$ objects showing a much faster decline in activation than $U$ objects. This suggests that not all aspects of RS in this study were directly related to repetition priming. A similar conclusion was reached by Sayers and Grill-Spector (2006) who found that the magnitude of RS across 7 stimulus repetitions of $\mathrm{F}$ objects was not correlated with the magnitude of RT priming across these repetitions in lateral occipital cortex and posterior fusiform gyrus. By contrast, van Turennout et al. (2003) observed qualitatively similar rates of RS across three stimulus presentations in occipital-temporal and inferior frontal cortices and repetition priming for $\mathrm{F}$ objects, suggestive of a direct relationship between these two processes. The reason for these differential findings is unclear, but it highlights need for further research on the precise relationship between RS, RE, and repetition priming. As pointed out by Henson (2003), neural repetition effects, as measured by fMRI, may reflect the average of several different, temporally separated repetition effects, some stemming from changes in the bottom-up analysis of stimuli and others from feedback from higher brain regions. It can be difficult, therefore, to interpret the absence of correlations between neural repetition effects, as measured by fMRI, and behavioral priming effects. For future studies on this topic, it would be fruitful to perform parallel or simultaneous fMRI and electrophysiological studies (i.e., EEG, ERP, MEG), which have a high temporal resolution (e.g., Fiebach et al., 2005).

In conclusion, this study showed that repetition priming of globally familiar and unfamiliar visual objects produces qualitatively different brain patterns of repetition effects. The data are consistent with the view that behavioral priming effects may reflect facilitation at multiple stages along the processing pathway between stimulus and response, including perceptual, semantic, decision, and motor processes. In addition, the present findings support the view that the repetition of globally unfamiliar objects can lead to the formation of new memory traces, as indexed by the enhancement in the neural response to repeated unfamiliar stimuli in some brain regions. These new memory traces did not appear to be of a perceptual nature, but may reflect the acquisition of more abstract, potentially lexical representations.

\section{Acknowledgments}

This work was supported by NIA grant RO1-AG16714 to Yaakov Stern.

We would like to thank Joseph Flynn and Elaine Gazes for research assistance.

\section{References}

Andersen, R.A., Snyder, L.H., Bradley, D.C., Xing, J., 1997. Multimodal representation of space in the posterior parietal cortex and its use in planning movements. Annu. Rev. Neurosci. 20, 303-330.

Astafiev, S.V., Shulman, G.L., Stanley, C.M., Snyder, A.Z., Van Essen, D.C., Corbetta, M., 2003. Functional organization of human intraparietal and frontal cortex for attending, looking, and pointing. J. Neurosci. 23 (11), 4689-4699.

Battaglia-Mayer, A., Caminiti, R., 2002. Optic ataxia as a result of the breakdown of the global tuning fields of parietal neurones. Brain 125 (Pt 2), 225-237.

Binder, J.R., Frost, J.A., Hammeke, T.A., Bellgowan, P.S., Rao, S.M., Cox, R.W., 1999. Conceptual processing during the conscious resting state. A functional MRI study. J. Cogn. Neurosci. 11 (1), 80-95.

Buchsbaum, B.R., Hickok, G., Humphries, C., 2001. Role of left posterior superior temporal gyrus in phonological processing for speech perception and production. Cogn. Sci. 25, 663-678.

Deeprose, C., Andrade, J., 2006. Is priming during anesthesia unconscious? Conscious. Cogn. 15 (1), 1-23.

Desimone, R., 1996. Neural mechanisms for visual memory and their role in attention. Proc. Natl. Acad. Sci. U. S. A. 93 (24), 13494-13499.

Dobbins, I.G., Schnyer, D.M., Verfaellie, M., Schacter, D.L., 2004. Cortical activity reductions during repetition priming can result from rapid response learning. Nature 428 (6980), 316-319.

Dietz, N.A., Jones, K.M., Gareau, L., Zeffiro, T.A., Eden, G.F., 2005. Phonological decoding involves left posterior fusiform gyrus. Hum. Brain Mapp. 26 (2), 81-93.

Etard, O., Mellet, E., Papathanassiou, D., Benali, K., Houde, O., Mazoyer, B., Tzourio-Mazoyer, N., 2000. Picture naming without Broca's and Wernicke's area. NeuroReport 11 (3), 617-622.

Fiebach, C.J., Gruber, T., Supp, G.G., 2005. Neuronal mechanisms of repetition priming in occipitotemporal cortex: spatiotemporal evidence from functional magnetic resonance imaging and electroencephalography. J. Neurosci. 25 (13), 3414-3422.

Gould, R.L., Brown, R.G., Owen, A.M., Bullmore, E.T., Howard, R.J., 2006. Task-induced deactivations during successful paired associates learning: an effect of age but not Alzheimer's disease. NeuroImage 31 (2), 818-831.

Grabowski, T.J., Damasio, H., Damasio, A.R., 1998. Premotor and prefrontal correlates f category-related lexical retrieval. NeuroImage 7 (3), 232-243.

Grill-Spector, K., Kushnir, T., Edelman, S., Avidan, G., Itzchak, Y., Malach, R., 1999. Differential processing of objects under various viewing conditions in the human lateral occipital complex. Neuron 24 (1), 187-203.

Gruber, T., Müller, M.M., 2005. Oscillatory brain activity dissociates between associative stimulus content in a repetition priming task in the human EEG. Cereb. Cortex 15 (1), 109-116.

Gusnard, D.A., Raichle, M.E., 2001. Searching for a baseline: functional imaging and the resting human brain. Nat. Rev., Neurosci. 2 (10), 685-694.

Habeck, C., Hilton, H.J., Zarahn, E., Brown, T., Stern, Y., 2006. An eventrelated fMRI study of the neural networks underlying repetition suppression and reaction time priming in implicit visual memory. Brain Res 1075 (1), 133-141.

Henson, R.N., 2003. Neuroimaging studies of priming. Prog. Neurobiol. 70 (1), 53-81. 
Henson, R.N., Rugg, M.D., 2003. Neural response suppression, haemodynamic repetition effects, and behavioural priming. Neuropsychologia 41 (3), 263-270

Henson, R., Shallice, T., Dolan, R., 2000. Neuroimaging evidence for dissociable forms of repetition priming. Science 287 (5456), 1269-1272.

Henson, R.N., Rylands, A., Ross, E., Vuilleumeir, P., Rugg, M.D., 2004. The effect of repetition lag on electrophysiological and haemodynamic correlates of visual object priming. NeuroImage 21 (4), 1674-1689.

Hilton, H.J., Pavlicic, T., Cooper, L.A., \& Stern, Y. (2006). Priming for impossible and for non-real objects is obtained in short term objectdecision implicit memory tasks. Unpublished manuscript.

Hirsch, J., Moreno, D.R., Kim, K.H., 2001. Interconnected large-scale systems for three fundamental cognitive tasks revealed by functional MRI. J. Cogn. Neurosci. 13 (3), 389-405.

Iacoboni, M., Woods, R.P., Mazziotta, J.C., 1998. Bimodal (auditory and visual) left frontoparietal circuitry for sensorimotor integration and sensorimotor learning. Brain 121 (Pt 11), 2135-2143.

Koutstaal, W., Wagner, A.D., Rotte, M., Maril, A., Buckner, R.L., Schacter, D.L., 2001. Perceptual specificity in visual object priming: functional magnetic resonance imaging evidence for a laterality difference in fusiform cortex. Neuropsychologia 39 (2), 184-199.

Krautzlis, R.J., 2005. The control of voluntary eye movements: new perspectives. Neuroscientist 11 (2), 124-137.

Lerner, Y., Hendler, T., Ben-Bashat, D., Harel, M., Malach, R., 2001. A hierarchical axis of object processing stages in the human visual cortex. Cereb. Cortex 11 (4), 287-297.

Li, L., Miller, E.K., Desimone, R., 1993. The representation of stimulus familiarity in anterior inferior temporal cortex. J. Neurophysiol. 69 (6), 1918-1929.

Liu, T., Cooper, L.A., 2001. The influence of task requirements on priming in object decision and matching. Mem. Cogn. 29 (6), 874-882.

Maccotta, L., Buckner, R.L., 2004. Evidence for neural effects of repetition that directly correlate with behavioral priming. J. Cogn. Neurosci. 16 (9), 1625-1632.

Marsolek, C.J., 1999. Dissociable neural subsystems underlie abstract and specific object recognition. Psychol. Sci. 10, 111-118.

McKiernan, K.A., Kaufman, J.N., Kucera-Thompson, J., Binder, J.R., 2003. A parametric manipulation of factors affecting task-induced deactivation in functional neuroimaging. J. Cogn. Neurosci. 15 (3), 394-408.

McKiernan, K.A., D’Angelo, B.R., Kaufman, J.N., Binder, J.R., 2006. Interrupting the "stream of consciousness": an fMRI investigation. NeuroImage 29 (4), 1185-1191.

Orfanidou, E., Marslen-Wilson, W.D., Davis, M.H., 2006. Neural response suppression predicts repetition priming of spoken words and pseudowords. J. Cogn. Neurosci. 18, 1237-1252.

Pourtois, G., Schwartz, S., Seghier, M.L., Lazeyras, F., Vuilleumier, P., 2005. Portraits or people? Distinct representations of face identity in the human visual cortex. J. Cogn. Neurosci. 17 (7), 1043-1057.

Pulvermüller, F., Huss, M., Kherif, F., Moscoso del Prado Martin, F., Hauk, O., Shtyrov, Y., 2006. Motor cortex maps articulatory features of speech sounds. Proc. Natl. Acad. Sci. U. S. A. 103 (20), 7865-7870.

Raichle, M.E., MacLeod, A.M., Snyder, A.Z., Powers, W.J., Gusnard, D.A., Shulman, G.L., 2001. A default mode of brain function. Proc. Natl. Acad. Sci. U. S. A. 98 (2), 676-682.

Reber, P.J., Gitelman, D.R., Parrish, T.B., Mesulam, M.M., 2005. Priming effects in the fusiform gyrus: changes in neural activity beyond the second presentation. Cereb. Cortex 15 (6), 787-795.

Ringo, J.L., 1996. Stimulus specific adaptation in inferior temporal and medial temporal cortex of the monkey. Behav. Brain Res. 76 (1-2), 191-197.

Roediger III, H.L., McDermott, K.B., 1993. Implicit memory in normal human subjects. In: Boller, F., Grafman, J. (Eds.), Handbook of Neuropsychology, vol. 8. Elsevier, Amsterdam, pp. 63-131.

Rushworth, M.F., Johansen-Berg, H., Gobel, S.M., Devlin, J.T., 2003. The left parietal and premotor cortices: motor attention and selection. NeuroImage 20 (Suppl. 1), S89-S100.

Sayres, R., Grill-Spector, K., 2006. Object-selective cortex exhibits performance-independent repetition suppression. J. Neurophysiol. 95 (2), 995-1007.

Schacter, D.L., Buckner, R.L., 1998. Priming and the brain. Neuron 20 (2), 185-195.

Schacter, D.L., Chiu, C.Y., Ochsner, K.N., 1993. Implicit memory: a selective review. Annu. Rev. Neurosci. 16, 159-182.

Schacter, D.L., Reiman, E., Uecker, A., Polster, M.R., Yun, L.S., Cooper, L.A., 1995. Brain regions associated with retrieval of structurally coherent visual information. Nature 376 (6541), 587-590.

Schnyer, D.M., Dobbins, I.G., Nicholls, L., Schacter, D.L., Verfaellie, M., 2006. Rapid response learning in amnesia: delineating associative learning components in repetition priming. Neuropsychologia 44 (1), 140-149.

Schubotz, R.I., von Cramon, D.Y., 2003. Functional-anatomical concepts of human premotor cortex: evidence from fMRI and PET studies. NeuroImage 20 (Suppl 1), S120-S131.

Simons, J.S., Koutstaal, W., Prince, S., Wagner, A.D., Schacter, D.L., 2003. Neural mechanisms of visual object priming: evidence for perceptual and semantic distinctions in fusiform cortex. NeuroImage 19 (3), 613-626.

Snodgrass, J.G., Vanderwart, M., 1980. A standardized set of 260 pictures: norms for name agreement, image agreement, familiarity, and visual complexity. J. Exp. Psychol. Hum. Learn 6 (2), 174-215.

Sobotka, S., Ringo, J.L., 1994. Stimulus specific adaptation in excited but not in inhibited cells in inferotemporal cortex of macaque. Brain Res. 646 (1), 95-99.

Soldan, A., Mangels, J.A., Cooper, L.A., 2006. Evaluating models of objectdecision priming: evidence from event-related potential repetition effects. J. Exper. Psychol., Learn., Mem., Cogn. 32 (2), 230-248.

Tanabe, H.C., Kato, M., Miyauchi, S., Hayashi, S., Yanagida, T., 2005. The sensorimotor transformation of cross-modal spatial information in the anterior intraparietal sulcus as revealed by functional MRI. Brain Res. Cogn. Brain Res. 22 (3), 385-396.

Thiel, C.M., Henson, R.N., Dolan, R.J., 2002. Scopolamine but not lorazepam modulates face repetition priming: a psychopharmacological fMRI study. Neuropsychopharmacology 27 (2), 282-292.

Tulving, E., Schacter, D.L., 1990. Priming and human memory systems. Science 247 (4940), 301-306.

van Turennout, M., Ellmore, T., Martin, A., 2000. Long-lasting cortical plasticity in the object naming system. Nat. Neurosci. 3 (12), 1329-1334.

van Turennout, M., Bielamowicz, L., Martin, A., 2003. Modulation of neural activity during object naming: effects of time and practice. Cereb. Cortex 13 (4), 381-391.

Vuilleumier, P., Henson, R.N., Driver, J., Dolan, R.J., 2002. Multiple levels of visual object constancy revealed by event-related fMRI of repetition priming. Nat. Neurosci. 5 (5), 491-499.

Wiggs, C.L., Martin, A., 1998. Properties and mechanisms of perceptual priming. Curr. Opin. Neurobiol. 8 (2), 227-233.

Wise, S.P., Boussaoud, D., Johnson, P.B., Caminiti, R., 1997. Premotor and parietal cortex: corticocortical connectivity and combinatorial computations. Annu. Rev. Neurosci. 20, 25-42.

Worsley, K.J., Marrett, S., Neelin, P., Vandal, A.C., Friston, K.J., Evans, A.C., 1996. A unified statistical approach for determining significant signals in images of cerebral activation. Hum. Brain Mapp. 4, 58-73.

Worsley, K.J., Poline, J.B., Friston, K.J., Evans, A.C., 1997. Characterizing the response of PET and $\mathrm{fMRI}$ data using multivariate linear models. NeuroImage 6 (4), 305-319.

Zago, L., Fenske, M.J., Aminoff, E., Bar, M., 2005. The rise and fall of priming: how visual exposure shapes cortical representations of objects. Cereb. Cortex 15 (11), 1655-1665.

Zarahn, E., Hilton, H.J., Abela, D., Stern, Y., 2004. Spatial Patterns of Object-Repetition Related Effects, 2004 Annual Cognitive Neuroscience Society Meeting, San Francisco.

Zarahn, E., Rakitin, B., Abela, D., Flynn, J., Stern, Y., 2005. Positive evidence against human hippocampal involvement in working memory maintenance of familiar stimuli. Cereb. Cortex 15 (3), 303-316.

Zarahn, E., Rakitin, B., Abela, D., Flynn, J., Stern, Y., 2007. Age-related changes in brain activation during a delayed item recognition task. Neurobiol. Aging 28 (5), 784-798. 